\title{
Characterizing Some Rings of Finite Order
}

\author{
Jutirekha Dutta, Dhiren Kumar Basnet and Rajat Kanti Nath
}

\begin{abstract}
In this paper, we compute the number of distinct centralizers of some classes of finite rings. We then characterize all finite rings with $n$ distinct centralizers for any positive integer $n \leq 5$. Further we give some connections between the number of distinct centralizers of a finite ring and its commutativity degree.
\end{abstract}

\section{Introduction}

Finite abelian groups have been completely characterized up to isomorphism for a long time but finite rings have yet to be characterized. The problem of characterizing finite rings up to isomorphism has received considerable attention in recent years (see $[2,8,10,12,13])$ starting from the works of Eldridge [11] and Raghavendran [15]. In this paper we characterize finite rings in terms of their number of distinct centralizers. Given a ring $R$ and an element $r \in R$, the subrings $C(r)=\{s \in R: r s=s r\}$ and $Z(R)=\{s \in R: r s=s r$ for all $r \in R\}$ are known as centralizer of $r$ in $R$ and center of $R$ respectively. We write $\operatorname{Cent}(R)$ to denote the set of all centralizers in $R$. Firstly we compute the order of $\operatorname{Cent}(R)$ for some classes of finite rings $R$. Motivated by the works of Belcastro and Sherman [3] and Ashrafi [1], we define $n$-centralizer ring for any positive integer $n$. A ring $R$ is said to be $n$-centralizer ring if $|\operatorname{Cent}(R)|=n$, for any positive integer $n$. We then characterize $n$-centralizer finite rings for all $n \leq 5$, adapting similar techniques that are used by Belcastro and Sherman [3] in order to characterize $n$-centralizer finite groups for $n \leq 5$. It is worth mentioning that 6,7 -centralizer finite rings have been characterized in [9].

Further, we conclude the paper by noting some interesting connections between $d(R)$ and $|\operatorname{Cent}(R)|$, where $d(R)$ is the probability that a randomly chosen pair of elements of $R$ commute. For any finite ring $R$ we have $d(R)=\frac{1}{|R|^{2}} \sum_{r \in R}|C(r)|$. This $d(R)$ is also known as commutativity 
degree or commuting probability of $R$ and it was introduced by MacHale [14] in the year 1976. Some characterizations of finite rings in terms of commutativity degree can be found in $[14,5,6]$.

Throughout the paper $R$ denotes a finite ring possibly non-associative and non-unital. For any subring $S$ of $R, R / S$ and $\frac{R}{S}$ denote the additive quotient group and $|R: S|$ denotes the index of the additive subgroup $S$ in the additive group $R$. Note that the isomorphisms considered are the additive group isomorphisms. Also for any two non-empty subsets $A$ and $B$ of a ring $R$, we write $A+B=\{a+b: a \in A, b \in B\}$. We shall use the fact that for any non-commutative ring $R$, the additive group $\frac{R}{Z(R)}$ is not a cyclic group (see [14, Lemma 1]).

\section{Some computations of $|\operatorname{Cent}(R)|$}

In this section, we compute $|\operatorname{Cent}(R)|$ for some classes of finite rings. However, first we prove some results which are useful for subsequent results as well as for the next sections.

Proposition 2.1. $R$ is a commutative ring if and only if $R$ is a 1-centralizer ring.

Proof. The proposition follows from the fact that a ring $R$ is commutative if and only if $C(r)=R$ for each $r \in R$.

Proposition 2.2. Let $R, S$ be two rings. Then

$$
\operatorname{Cent}(R \times S)=\operatorname{Cent}(R) \times \operatorname{Cent}(S)
$$

Proof. It can be easily seen that $C((r, s))=C(r) \times C(s)$ for any $r \in R$ and $s \in S$. This proves the proposition.

The following lemmas play an important role in finding lower bound of $|\operatorname{Cent}(R)|$ for any non-commutative ring $R$.

Lemma 2.1. Let $R$ be a ring. Then $Z(R)$ is the intersection of all centralizers in $R$.

Proof. It is clear that $Z(R) \subseteq \underset{r \in R}{\cap} C(r)$. Now, for any $s \in \underset{r \in R}{\cap} C(r)$ we have $r s=s r$ for all $r \in R$. Therefore $s \in Z(R)$. Hence the lemma follows.

Lemma 2.2. If $R$ is a ring, then $R$ is the union of centralizers of all non-central elements of $R$.

Proof. It is clear that $\underset{r \in R-Z(R)}{\cup} C(r) \subseteq R$. Again, for any $s \in Z(R)$, we have by Lemma 2.1, $s \in C(r)$ for all $r \in R$. So $s \in \underset{r \in R-Z(R)}{\cup} C(r)$. Also for any $s \in R-Z(R)$, we have $s \in C(s)$ and so $s \in \underset{r \in R-Z(R)}{\cup} C(r)$. Hence the lemma follows. 
Lemma 2.3. A ring $R$ cannot be written as a union of two of its proper subrings.

Proof. The lemma follows from the well-known fact that a group can not be written as a union of two of its proper subgroups.

Theorem 2.1. For any non-commutative ring $R,|\operatorname{Cent}(R)| \geq 4$.

Proof. Since $R$ is non-commutative, so $|\operatorname{Cent}(R)| \geq 2$. If $|\operatorname{Cent}(R)|=2$, then, by Lemma 2.2, $R$ is equal to a proper subset of itself, which is not possible. Also by Lemma $2.3,|\operatorname{Cent}(R)| \neq 3$. Hence the theorem follows.

Note that the ring $R=\left\{\left[\begin{array}{ll}0 & 0 \\ 0 & 0\end{array}\right],\left[\begin{array}{ll}1 & 0 \\ 1 & 0\end{array}\right],\left[\begin{array}{ll}0 & 1 \\ 0 & 1\end{array}\right],\left[\begin{array}{ll}1 & 1 \\ 1 & 1\end{array}\right]\right\}$, where $0,1 \in \mathbb{Z}_{2}$, has 4 distinct centralizers. So the above result is the best one possible.

At this point, the following question, similar to the question posed by Belcastro and Sherman $[3$, p. 371], arises naturally.

Question 2.2. Does there exist an $n$-centralizer ring for any positive integer $n \neq 2,3$ ? Can we characterize an $n$-centralizer ring?

The following results show the existence of $n$-centralizer rings for some values of $n$.

Proposition 2.3. There exists a $(p+2)$-centralizer ring for any prime $p$.

Proof. We consider the ring

$$
R=\left\{\left[\begin{array}{ll}
a & b \\
0 & 0
\end{array}\right]: a, b \in \mathbb{Z}_{p}\right\}
$$

For any element $\left[\begin{array}{ll}x & y \\ 0 & 0\end{array}\right]$ of $C\left(\left[\begin{array}{ll}a & b \\ 0 & 0\end{array}\right]\right)$ we have $x b-a y=0$.

Clearly, $C\left(\left[\begin{array}{ll}0 & 0 \\ 0 & 0\end{array}\right]\right)=R$. Using simple calculations, we have for any $a \neq 0$ and $l \in \mathbb{Z}_{p}$, $C\left(\left[\begin{array}{ll}a & 0 \\ 0 & 0\end{array}\right]\right)=\left\{\left[\begin{array}{ll}x & 0 \\ 0 & 0\end{array}\right]: x \in \mathbb{Z}_{p}\right\}$ and $C\left(\left[\begin{array}{cc}l a & a \\ 0 & 0\end{array}\right]\right)=\left\{\left[\begin{array}{cc}l x & x \\ 0 & 0\end{array}\right]: x \in \mathbb{Z}_{p}\right\}$. Hence $|\operatorname{Cent}(R)|=p+2$.

The above proposition is a particular case of the following theorem.

Theorem 2.3. Let $R$ be a non-commutative ring of order $p^{2}$, where $p$ is a prime. Then $|\operatorname{Cent}(R)|=$ $p+2$. 
Proof. For any $x \in R-Z(R)$, we consider $C(x)$. As $C(x)$ is an additive subgroup of $R$ we have $|C(x)|=1, p$ or $p^{2}$. Clearly, $|C(x)| \neq 1, p^{2}$, as $x, 0_{R} \in C(x)$ and $R$ is non-commutative, where $0_{R}$ is the additive identity in $R$. Hence $C(x)$ is additive cyclic group of order $p$ and so $Z(R)=\left\{0_{R}\right\}$.

Let $x, y \in R-Z(R)$. If there exists an element $t\left(\neq 0_{R}\right) \in C(x) \cap C(y)$ then $C(x)=C(y)$, as $C(x), C(y)$ are additive cyclic groups of order $p$. Thus for any $x, y \in R-Z(R)$ we have either $C(x) \cap C(y)=\left\{0_{R}\right\}$ or $C(x)=C(y)$. Therefore the number of centralizers of non-central elements is

$$
\frac{|R|-|Z(R)|}{p-1}=\frac{p^{2}-1}{p-1}=p+1 .
$$

Hence $|\operatorname{Cent}(R)|=p+2$.

Theorem 2.4. Let $p$ be a prime number and $R$ be a non-commutative ring of order $p^{3}$ with unity $1_{R}$. Then $|\operatorname{Cent}(R)|=p+2$.

Proof. Let $x$ be an arbitrary element of $R-Z(R)$. Then $C(x)$ is an additive subgroup of $R$ and so $|C(x)|=1, p, p^{2}$ or $p^{3}$. Here $|C(x)| \neq 1, p^{3}$ as $x, 0_{R} \in C(x)$, where $0_{R}$ is the additive identity in $R$ and $R$ is non-commutative. If $|C(x)|=p$ then $|Z(R)|=1$, which is not possible as $0_{R}, 1_{R} \in Z(R)$. So $|C(x)|=p^{2}$ and this gives $|Z(R)|=p$.

Now, we suppose that $y \in R-Z(R)$ and $y \in C(x)$. Let $z \in C(x)$ be an arbitrary element. We know that $Z(R) \subset Z(C(x))$ and so $|Z(C(x))|>1$. Therefore $|C(x): Z(C(x))|=1$ or $p$ and so $C(x)$ is commutative. Thus $z \in C(y)$, as $y \in C(x)$. So $C(x) \subseteq C(y)$. Also $|C(x)|=|C(y)|$. Hence, $C(x)=C(y)$; and if $y \notin C(x)$ then $C(x) \cap C(y)=Z(R)$. Therefore the number of centralizers of non-central elements of $R$ is

$$
\frac{|R|-|Z(R)|}{|C(x)|-|Z(R)|}=\frac{p^{3}-p}{p^{2}-p}=p+1 .
$$

Thus $|\operatorname{Cent}(R)|=p+2$.

As an application of the above theorem, it follows that the ring

$$
R=\left\{\left[\begin{array}{ll}
a & b \\
0 & c
\end{array}\right]: a, b, c \in \mathbb{Z}_{p}\right\}
$$

having order $p^{3}$ is a $(p+2)$-centralizer ring. The following theorem, which is a generalization of Theorem 2.3, gives another class of $(p+2)$-centralizer rings .

Theorem 2.5. Let $R$ be a ring and $\frac{R}{Z(R)} \cong \mathbb{Z}_{p} \times \mathbb{Z}_{p}$, where $p$ is a prime. Then $|\operatorname{Cent}(R)|=p+2$. 
Proof. We write $Z:=Z(R)$. Since $R / Z \cong \mathbb{Z}_{p} \times \mathbb{Z}_{p}$ we have

$$
\frac{R}{Z}=\langle Z+a, Z+b: p(Z+a)=p(Z+b)=Z ; a, b \in R\rangle .
$$

If $S / Z$ is additive non-trivial subgroup of $R / Z$ then $|S / Z|=p$. Therefore any additive proper subgroup of $R$ properly containing $Z$ has $p$ disjoint right cosets. Hence the proper additive subgroups of $R$ properly containing $Z$ are

$$
\begin{aligned}
& S_{m}=Z \cup(Z+(a+m b)) \cup(Z+2(a+m b)) \cup \cdots \cup(Z+(p-1)(a+m b)), \\
& \quad \text { where } 1 \leq m \leq(p-1), \\
& S_{p}=Z \cup(Z+a) \cup(Z+2 a) \cup \cdots \cup(Z+(p-1) a) \text { and } \\
& S_{p+1}=Z \cup(Z+b) \cup(Z+2 b) \cup \cdots \cup(Z+(p-1) b) .
\end{aligned}
$$

Now for any $x \in R-Z$, we have $Z+x$ is equal to $Z+k$ for some $k \in\{m a, m b, a+m b, 2(a+$ $m b), \ldots,(p-1)(a+m b): 1 \leq m \leq(p-1)\}$. Therefore $C(x)=C(k)$. Again, let $y \in S_{j}-Z$ for some $j \in\{1,2, \ldots,(p+1)\}$, then $C(y) \neq S_{q}$, where $1 \leq q(\neq j) \leq(p+1)$. Thus $C(y)=S_{j}$. Hence $|\operatorname{Cent}(R)|=p+2$.

Further, we have the following theorem analogous to Lemma 2.7 of [1, p. 142].

Theorem 2.6. Let $R$ be a non-commutative ring whose order is a power of a prime $p$. Then $|\operatorname{Cent}(R)|$ $\geq p+2$, and equality holds if and only if $\frac{R}{Z(R)} \cong \mathbb{Z}_{p} \times \mathbb{Z}_{p}$.

Proof. Let $R$ be a non-commutative ring whose order is a power of a prime $p$. Suppose $k=$ $|\operatorname{Cent}(R)|$. Let $A_{1}, \ldots, A_{k}$ be the distinct centralizers of $R$ such that $\left|A_{1}\right| \geq \cdots \geq\left|A_{k}\right|$ and $A_{1}=R$. So $R=\bigcup_{i=2}^{k} A_{i}$ and by Cohn's Theorem in [7, p. 44], we have $|R| \leq \sum_{i=3}^{k}\left|A_{i}\right|$ (as $A_{i}$ 's are additive groups). Also $\left|A_{i}\right| \leq \frac{|R|}{p}$, where $i \neq 1$. Hence

$$
|R| \leq \underbrace{\frac{|R|}{p}+\cdots+\frac{|R|}{p}}_{(k-2)-\text { times }}
$$

which implies $|R| \leq(k-2) \frac{|R|}{p}$ and so $k \geq p+2$. That is $|\operatorname{Cent}(R)| \geq p+2$.

For the equality, if $\frac{R}{Z(R)} \cong \mathbb{Z}_{p} \times \mathbb{Z}_{p}$ then by Theorem 2.5, we have $|\operatorname{Cent}(R)|=p+2$. Conversely, we assume that $l=|\operatorname{Cent}(R)|=p+2$. Suppose $A_{1}, A_{2}, \ldots, A_{l}$ are distinct centralizers of $R$ such that $\left|A_{1}\right| \geq \cdots \geq\left|A_{l}\right|$ and $A_{1}=R$. So $R=\bigcup_{i=2}^{l} A_{i}$ and by Cohn's Theorem in [7, p. 
44], we have $|R| \leq \sum_{i=3}^{l}\left|A_{i}\right|$. Also $\left|A_{i}\right| \leq \frac{|R|}{p}$, where $i \neq 1$. Suppose, there exists an $A_{i}$ such that $\left|A_{i}\right|<\frac{|R|}{p}$ for $3 \leq i \leq l$ then

$$
|R|<\underbrace{\frac{|R|}{p}+\cdots+\frac{|R|}{p}}_{(l-2) \text {-times }}=(l-2) \frac{|R|}{p}=|R|,
$$

a contradiction. Hence $\left|A_{3}\right|=\frac{|R|}{p}, \ldots,\left|A_{l}\right|=\frac{|R|}{p}$. Also $\left|A_{2}\right| \geq \cdots \geq\left|A_{l}\right|$, so $\left|A_{i}\right|=\frac{|R|}{p}$, where $2 \leq i \leq l$. Hence $\sum_{i=3}^{l}\left|A_{i}\right|=(l-2) \frac{|R|}{p}=|R|$. Therefore $\sum_{i=3}^{l}\left|A_{i}\right|=|R|$ if and only if $A_{2}+A_{m}=R$, for all $m \neq 2$ and $A_{k} \cap A_{l} \subseteq A_{2}$ for all $k \neq l$ (By Cohn's Theorem in [7, p. 44]). Interchanging $A_{i}$ 's we have $A_{2} \cap A_{3}=Z(R)$. Thus

$$
|R|=\left|A_{2}+A_{3}\right|=\frac{\left|A_{2}\right|\left|A_{3}\right|}{\left|A_{2} \cap A_{3}\right|}=\frac{|R|^{2}}{p^{2}|Z(R)|}
$$

which gives $|R: Z(R)|=p^{2}$. Hence $\frac{R}{Z(R)} \cong \mathbb{Z}_{p} \times \mathbb{Z}_{p}$, since $R$ is non-commutative. This completes the proof.

\section{4-centralizer rings}

In this section, we give a characterization of finite 4-centralizer rings analogous to Theorem 2 of [3, p. 367]. The following lemma is useful in characterization of 4-centralizer rings.

Lemma 3.1. Let $R$ be a 4-centralizer finite ring. Then at least one of the centralizers of non-central elements has index 2 in $R$.

Proof. Let $A, B, C$ be the three proper centralizers of $R$. Suppose none of $A, B, C$ has index 2, that is $|R: A| \geq 3,|R: B| \geq 3,|R: C| \geq 3$. Then as $R=A \cup B \cup C$, we have

$$
|R| \leq|A|+|B|+|C|-2|Z(R)| \leq \frac{|R|}{3}+\frac{|R|}{3}+\frac{|R|}{3}-2|Z(R)|<|R|,
$$

which is a contradiction. Hence the lemma follows.

We have the following characterization of finite 4-centralizer rings.

Theorem 3.1. Let $R$ be a non-commutative finite ring. Then $|\operatorname{Cent}(R)|=4$ if and only if $\frac{R}{Z(R)} \cong$ $\mathbb{Z}_{2} \times \mathbb{Z}_{2}$. 
Proof. If $\frac{R}{Z(R)} \cong \mathbb{Z}_{2} \times \mathbb{Z}_{2}$ then by Theorem 2.5, we have $|\operatorname{Cent}(R)|=4$.

Conversely, let $|\operatorname{Cent}(R)|=4$ then $R$ has exactly four distinct centralizers, say $R, A, B, C$ where $A, B, C$ are three distinct centralizers of non-central elements of $R$.

By Lemma 2.3, $R$ can not be written as the union of two of its proper subrings of $R$. Therefore we may choose $a \in A-(B \cup C), b \in B-(C \cup A)$ and $c \in C-(A \cup B)$ respectively. It can be easily seen that $C(a)=A, C(b)=B$ and $C(c)=C$. By Lemma 3.1, at least one of the centralizers $A, B, C$, say $A$ has index 2 in $R$, that is $|R: A|=2$.

Now, let $x \in(A \cap B)-Z(R)$ then $C(x) \neq R$. If $C(x)=A$ then $a, b \in C(x)$. So, $C(x) \neq A$. Similarly it can be seen that $C(x) \neq B$. If $C(x)=C$ then $x \in A \cap B \cap C=Z(R)$ (using Lemma 2.1), which is a contradiction. Therefore $|\operatorname{Cent}(R)|$ must be at least 5 , which is again a contradiction. So $A \cap B=A \cap B \cap C=Z(R)$. Similarly it can be seen that $B \cap C=$ $Z(R), A \cap C=Z(R)$. Again $A, B, C$ are additive subgroups of $R$, therefore

$$
|R| \geq|A+B|=\frac{|A||B|}{|A \cap B|}=\frac{|A||B|}{|Z(R)|}
$$

which gives $|B| \leq 2|Z(R)|$. Since $Z(R) \subset B$, so $\frac{|B|}{2} \leq|Z(R)|<|B|$. Hence $|B|=2|Z(R)|$. Similarly $|C|=2|Z(R)|$. Therefore

$$
|R|=|A|+|B|+|C|-2|Z(R)|=\frac{|R|}{2}+2|Z(R)|
$$

which gives $|R: Z(R)|=4$ and hence $\frac{R}{Z(R)} \cong \mathbb{Z}_{2} \times \mathbb{Z}_{2}$.

\section{5-centralizer rings}

In this section, we give a characterization of finite 5 -centralizer rings analogous to Theorem 4 of [3, p. 369]. The following lemmas are useful in this regard.

Lemma 4.1. Let $R$ be a ring and $R=A \cup B \cup C$, where $A, B, C$ are the proper distinct subrings. We put $K=A \cap B \cap C, L=A \cap B-K, M=A \cap C-K, N=B \cap C-K$ and $A^{\prime}=A-(B \cup C)$, $B^{\prime}=B-(A \cup C), C^{\prime}=C-(A \cup B)$. Then

(a) $L=M=N=\emptyset$,

(b) $A^{\prime}+B^{\prime} \subseteq C^{\prime}, B^{\prime}+C^{\prime} \subseteq A^{\prime}$ and $C^{\prime}+A^{\prime} \subseteq B^{\prime}$,

(c) $A^{\prime}+A^{\prime} \subseteq K, B^{\prime}+B^{\prime} \subseteq K$ and $C^{\prime}+C^{\prime} \subseteq K$,

(d) $|R: K|=4$. 
Proof. (a) We consider $l \in L$ and $c^{\prime} \in C^{\prime}$. Then $c^{\prime}+l \in A$ or $B$ or $C$. If $c^{\prime}+l \in A$ then $c^{\prime}+l+(-l)=c^{\prime} \in A$, a contradiction. If $c^{\prime}+l \in B$ then $c^{\prime}+l+(-l)=c^{\prime} \in B$, a contradiction. If $c^{\prime}+l \in C$ then $\left(-c^{\prime}\right)+c^{\prime}+l=l \in C$, a contradiction. Since $C^{\prime} \neq \emptyset$, we must have $L=\emptyset$. Similarly $M=N=\emptyset$.

(b) Let $a^{\prime} \in A^{\prime}$, then $a^{\prime} \in A \Rightarrow-a^{\prime} \in A \Rightarrow-a^{\prime} \in K$ or $A^{\prime}$. If $-a^{\prime} \in K$ then $a^{\prime} \in K$, a contradiction. Hence $-a^{\prime} \in A^{\prime}$. Similarly if $b^{\prime} \in B^{\prime}$ then $-b^{\prime} \in B^{\prime}$ and if $c^{\prime} \in C^{\prime}$ then $-c^{\prime} \in C^{\prime}$. Suppose $a^{\prime} \in A^{\prime}, b^{\prime} \in B^{\prime}$ then $a^{\prime}+b^{\prime} \in K$ or $A^{\prime}$ or $B^{\prime}$ or $C^{\prime}$. If $a^{\prime}+b^{\prime} \in A^{\prime} \subseteq A$ then $b^{\prime}=-a^{\prime}+a^{\prime}+b^{\prime} \in A$, a contradiction. If $a^{\prime}+b^{\prime} \in B^{\prime} \subseteq B$, then $a^{\prime}=a^{\prime}+b^{\prime}+\left(-b^{\prime}\right) \in B$, a contradiction. If $a^{\prime}+b^{\prime} \in K$, then $a^{\prime}+b^{\prime} \in A$, a contradiction. Hence $a^{\prime}+b^{\prime} \in C^{\prime}$. Thus $A^{\prime}+B^{\prime} \subseteq C^{\prime}$. Similarly it can be seen that $B^{\prime}+C^{\prime} \subseteq A^{\prime}$ and $C^{\prime}+A^{\prime} \subseteq B^{\prime}$.

(c) Let $a^{\prime}, a_{1}{ }^{\prime} \in A^{\prime} \subseteq A$. So $a^{\prime}+a_{1}{ }^{\prime} \in A \Rightarrow a^{\prime}+a_{1}{ }^{\prime} \in A^{\prime}$ or $K$. Let $a^{\prime}+a_{1}{ }^{\prime} \in A^{\prime}$. We consider $b^{\prime}+a^{\prime}+a_{1}{ }^{\prime}$, for some $b^{\prime} \in B^{\prime}$. Then by second part we have $b^{\prime}+\left(a^{\prime}+a_{1}{ }^{\prime}\right) \in C^{\prime}$ and $\left(b^{\prime}+a^{\prime}\right)+a_{1}{ }^{\prime} \in B^{\prime}$. So $b^{\prime}+a^{\prime}+a_{1}{ }^{\prime} \in B^{\prime} \cap C^{\prime}$, a contradiction. Similarly we can show the other two.

(d) From part (a), we have $R=K \cup A^{\prime} \cup B^{\prime} \cup C^{\prime}$. Let $k+a^{\prime} \in K+a^{\prime}$ where $k \in K, a^{\prime} \in A^{\prime}$ then $k+a^{\prime} \in A=K \cup A^{\prime}$. If $k+a^{\prime} \in K$ then $a^{\prime} \in K$, a contradiction. So $K+a^{\prime} \subseteq A^{\prime}$. Again $x^{\prime} \in A^{\prime}$ gives $x^{\prime}+\left(-a^{\prime}\right) \in K$ (by part (c)). So, $x^{\prime} \in K+a^{\prime}$. Hence $K+a^{\prime}=A^{\prime}$. Similarly it can be seen that $K+b^{\prime}=B^{\prime}, K+c^{\prime}=C^{\prime}$, where $b^{\prime} \in B^{\prime}, c^{\prime} \in C^{\prime}$. Therefore $|R: K|=4$.

Lemma 4.2. Let $R$ be a 5-centralizer finite ring and $A, B, C, D$ be the four proper centralizers of R. Then

(a) $|R|=|A|+|B|+|C|+|D|-3|Z(R)|$.

(b) If $S$ and $T$ are distinct proper centralizers of $R$, then

$$
\frac{|S||T|}{|R|} \leq|Z(R)| \leq \frac{|R|}{6} .
$$

Proof. Let $a \in A-(B \cup C), b \in B-(A \cup C)$ and $c \in C-(A \cup B)$. Suppose there does not exist any $a \in A-(B \cup C)$ such that $C(a)=A$. Then $C(a)=D$ for all $a \in A-(B \cup C)$. Therefore $A-(B \cup C) \subseteq D-(B \cup C)$. Interchanging the roles of $A$ and $D$ we get $A-(B \cup C)=D-(B \cup C)$, which gives $A \cup B \cup C=D \cup B \cup C=R$. Again, by Lemma 4.1(a), we have $B \cap C=C \cap D$ and so $Z(R)=A \cap B \cap C$. Therefore, by Lemma 4.1(d), we have $R / Z(R) \cong \mathbb{Z}_{2} \times \mathbb{Z}_{2}$. This gives $|\operatorname{Cent}(R)|=4$, contradiction. Hence $C(a)=A$. Similarly $C(b)=B$ and $C(c)=C$.

(a) Let us assume without loss of generality that $D$ is a subset of $A \cup B \cup C$. Then $R=A \cup B \cup$ $C \cup D=A \cup B \cup C$. Now, by Lemma 4.1, we have $|R: K|=4$ where $K=A \cap B \cap C=Z(R)$. Thus by Theorem 3.1, $|\operatorname{Cent}(R)|=4$, which is a contradiction. Therefore no one of $A, B, C$ or $D$ is contained in the union of the other three. 
Let $r \in(A \cap B)-(C \cup D)$ then $r \in C(a) \cap C(b)$ which gives $a, b \in C(r)$. But $a \notin C(b)$, so $C(r) \neq A, B$. Again $r \notin C, D$; so $C(r) \neq C, D$. Also $C(r) \neq R$, since $r \in R-Z(R)$. Therefore $|\operatorname{Cent}(R)|$ must be at least 6 , a contradiction. Hence $(A \cap B)-(C \cup D)=\emptyset$. This shows that no element of $R$ is in exactly two proper centralizers.

Let $r \in(A \cap B \cap C)-D$ then $r \in C(a) \cap C(b) \cap C(c)$. Therefore $a, b, c \in C(r)$. But $b \notin C(a), c \notin C(b)$. So $C(r) \neq A, B, C$. Also $C(r) \neq D, R$; as $r \notin D$ and $r \notin Z(R)$. Therefore $|\operatorname{Cent}(R)|$ must be at least 6 , a contradiction. Hence $A \cap B \cap C-D=\emptyset$. Thus no element of $R$ is in exactly three proper centralizers.

From above, it can be seen clearly that

$$
|R|=|A \cup B \cup C \cup D|=|A|+|B|+|C|+|D|-3|Z(R)| .
$$

(b) Note that for any two proper centralizers $S$ and $T$ of $R$ we have $S \cap T=Z(R)$, since no element of $R$ is in exactly two as well as three proper centralizers. Also any proper centralizers of $R$ are additive subgroups of $R$, so $\frac{|S||T|}{|S+T|}=|S \cap T|=|Z(R)|$. Since $S+T \subseteq R$ we have $|Z(R)| \geq \frac{|S||T|}{|R|}$.

Again by part (a),

$$
\begin{aligned}
& |R|=|A|+|B|+|C|+|D|-3|Z(R)| \\
& \quad \geq 2|Z(R)|+2|Z(R)|+2|Z(R)|+2|Z(R)|-3|Z(R)| .
\end{aligned}
$$

Thus $|R: Z(R)| \geq 5$. If $|R: Z(R)|=5$ then $\frac{R}{Z(R)} \cong \mathbb{Z}_{5}$, a contradiction. Therefore $|Z(R)| \leq$ $\frac{|R|}{6}$. So, $\frac{|S||T|}{|R|} \leq|Z(R)| \leq \frac{|R|}{6}$.

We would like to mention here that the group theoretic analogues of Lemma 4.1 and Lemma 4.2 can be found in [4, p. 52-53] and [3, p. 370] respectively. Now we prove the main theorem of this section which characterizes finite 5 -centralizer rings.

Theorem 4.1. Let $R$ be a finite ring. Then $|\operatorname{Cent}(R)|=5$ if and only if $\frac{R}{Z(R)} \cong \mathbb{Z}_{3} \times \mathbb{Z}_{3}$.

Proof. Let $\frac{R}{Z(R)} \cong \mathbb{Z}_{3} \times \mathbb{Z}_{3}$, then by Theorem 2.5, we get $|\operatorname{Cent}(R)|=5$.

Conversely, let $|\operatorname{Cent}(R)|=5$. Let $A, B, C, D$ be the four proper centralizers of $R$. Then by Lemma 4.2(b), $\frac{|A||B|}{|R|} \leq|Z(R)| \leq \frac{|R|}{6}$. Our aim is to get more near lower bound for $|Z(R)|$. We may assume without loss of generality that $|A| \geq|B| \geq|C| \geq|D|$. Suppose $|A|<\frac{|R|}{3}$, as $1<$ $|A| \leq \frac{|R|}{2}$. That is $|A| \leq \frac{|R|}{4}$. Now by Lemma 4.2(a), $|R| \leq|R|-3|Z(R)|<|R|$, a contradiction. Hence $|A|=\frac{|R|}{2}$ or $|A|=\frac{|R|}{3}$. If $|A|=\frac{|R|}{2}$, then $|R|=|A|+|B|+|C|+|D|-3|Z(R)|$ gives $\frac{|R|}{2}<|B|+|C|+|D|$ and so $\frac{|R|}{6}<|B|$. Also, applying Lemma 4.2(b) on $A$ and $B$ we have $\frac{|R|}{6}<|B| \leq \frac{|R|}{3}$. So $|B|$ is one of $\frac{|R|}{3}, \frac{|R|}{4}$ or $\frac{|R|}{5}$. Reapplying Lemma 4.2(b) on $A$ and $B$ we have,

$$
\frac{|A||B|}{|R|} \leq|Z(R)| \leq \frac{|R|}{6}
$$


which gives $\frac{|R|}{10} \leq|Z(R)| \leq \frac{|R|}{6}$. Thus $|Z(R)|$ is one of $\frac{|R|}{6}, \frac{|R|}{7}, \frac{|R|}{8}, \frac{|R|}{9}$ or $\frac{|R|}{10}$. Let $|Z(R)|=$ $\frac{|R|}{7}, \frac{|R|}{9}$ then 2 divides 7 and 9 , which is not possible. If $|Z(R)|=\frac{|R|}{6}$ then $\frac{R}{Z(R)} \cong \mathbb{Z}_{6}$, a contradiction. Let $|Z(R)|=\frac{|R|}{8}$ then $\frac{|R|}{8}$ divides $|B|$. If $|B|=\frac{|R|}{3}, \frac{|R|}{5}$ then 3,5 divides 8 , a contradiction. Therefore $|B|=\frac{|R|}{4}$. By Lemma 4.2(a), we have $\frac{5|R|}{8}=|C|+|D|$. Also $|B| \geq|C| \geq|D|$. So $|C|+|D| \leq \frac{|R|}{2}<\frac{5|R|}{8}=|C|+|D|$, a contradiction. If $|Z(R)|=\frac{|R|}{10}$, then $\frac{|R|}{10}$ divides $|B|$. If $|B|=\frac{|R|}{3}, \frac{|R|}{4}$ then 3,4 divides 10 , a contradiction. Therefore $|B|=\frac{|R|}{5}$. Now Lemma 4.2(a) gives, $|C|+|D|=\frac{6|R|}{10}$. Also $|B| \geq|C| \geq|D|$, therefore $|C|+|D| \leq \frac{2|R|}{5}<\frac{6|R|}{10}=|C|+|D|$, a contradiction.

If $|A|=\frac{|R|}{3}$ then Lemma 4.2(a) gives, $\frac{2|R|}{3}<|B|+|C|+|D|$ which gives $\frac{2|R|}{3}<3|B|$ and so $|B| \geq \frac{|R|}{4}$. Also $|A| \geq|B|$, so $|B|=\frac{|R|}{3}$ or $\frac{|R|}{4}$. Again, applying Lemma 4.2(b) on $A$ and $B$ we get,

$$
\frac{|A||B|}{|R|} \leq|Z(R)| \leq \frac{|R|}{6}
$$

which gives $\frac{|R|}{12} \leq|Z(R)| \leq \frac{|R|}{6}$. Therefore $|Z(R)|$ is one of $\frac{|R|}{6}, \frac{|R|}{7}, \frac{|R|}{8}, \frac{|R|}{9}, \frac{|R|}{10}, \frac{|R|}{11}$ or $\frac{|R|}{12}$. Now if $|Z(R)|=\frac{|R|}{7}, \frac{|R|}{8}, \frac{|R|}{10}, \frac{|R|}{11}$ then 3 divides $7,8,10,11$, a contradiction. Let $|Z(R)|=\frac{|R|}{6}$ then as above we get a contradiction. Let $|Z(R)|=\frac{|R|}{9}$ then $\frac{R}{Z(R)} \cong \mathbb{Z}_{3} \times \mathbb{Z}_{3}$. Let $|Z(R)|=\frac{|R|}{12}$ and $|B|=\frac{|R|}{3}$ then applying Lemma $4.2(\mathrm{~b})$ on $A$ and $B$ we have, $\frac{|R|}{9} \leq \frac{|R|}{12}$, a contradiction. If $|B|=\frac{|R|}{4}$ then Lemma 4.2(a) gives, $|C|+|D|=\frac{4|R|}{6}$. Also $|C|,|D| \leq \frac{|R|}{4}$, so $|C|+|D| \leq$ $\frac{3|R|}{6}<\frac{4|R|}{6}=|C|+|D|$, which is not possible. Hence $\frac{R}{Z(R)} \cong \mathbb{Z}_{3} \times \mathbb{Z}_{3}$.

\section{Relation between $|\operatorname{Cent}(R)|$ and $d(R)$}

Note that $d(R)=1$ if and only if $R$ is commutative. Therefore, by Proposition 2.1, we have $|\operatorname{Cent}(R)|=1$ if and only if $d(R)=1$. By Theorem 3.1 and Theorem 1 of [14, p. 31], we have the following result.

Proposition 5.1. Let $R$ be a non-commutative finite ring. Then $|\operatorname{Cent}(R)|=4$ if and only if $d(R)=\frac{5}{8}$.

In $[14$, p. 31$]$, MacHale also proved the following theorem:

Theorem 5.1. Let $R$ be a non-commutative finite ring and $p$ the smallest prime dividing the order of $R$. Then $d(R) \leq \frac{1}{p^{3}}\left(p^{2}+p-1\right)$, with equality if and only if $|R: Z(R)|=p^{2}$.

Now by Theorem 2.5 and Theorem 5.1, we have the following interesting connection between $d(R)$ and $|\operatorname{Cent}(R)|$.

Proposition 5.2. Let $R$ be a non-commutative finite ring and $p$ the smallest prime dividing the order of $R$. If $d(R)=\frac{1}{p^{3}}\left(p^{2}+p-1\right)$ then $|\operatorname{Cent}(R)|=p+2$. 
We conclude the paper by noting that the converse of Proposition 5.2 holds for some finite non-commutative rings. In particular, by Theorem 2.6 and Theorem 5.1, we have the following result.

Proposition 5.3. Let $R$ be a non-commutative ring whose order is a power of a prime $p$. If $|\operatorname{Cent}(R)|$ $=p+2$ then $d(R)=\frac{1}{p^{3}}\left(p^{2}+p-1\right)$.

\section{Acknowledgments}

The authors would like to thank the referee for his/her valuable comments and suggestions. The first author is grateful to the Department of Mathematical Sciences, Tezpur University for its support while this investigation was carried out as a part of her Ph. D. thesis.

\section{References}

[1] A. R. Ashrafi, On finite groups with a given number of centralizers, Algebra Colloq., 7 (2000), 139-146.

[2] M. Behboodi, R. Beyranvand, A. Hashemi and H. Khabazian, Classification of finite rings: theory and algorithm, Czechoslovak Math. J., 64 (2014), 641-658.

[3] S. M. Belcastro and G. J. Sherman, Counting centralizers in finite groups, Math. Magazine, 67 (1994), 366-374.

[4] M. Bruckheimer, A. C. Bryan and A. Muir, Groups which are the union of three subgroups, Amer. Math. Monthly, 77 (1970), 52-57.

[5] S. M. Buckley, D. MacHale and A. Ní Shé, Finite rings with many commuting pairs of elements. Available from: http://archive.maths.nuim.ie/staff/sbuckley/Papers/ bms.pdf .

[6] S. M. Buckley and D. MacHale, Contrasting the commuting probabilities of groups and rings. Available from: http://archive.maths.nuim.ie/staff/sbuckley/Papers/ bm_g-vs-r.pdf.

[7] J. H. E. Cohn, On $n$-sum groups, Math. Scand., 75 (1994), 44-58.

[8] C. J. Chikunji, A Classification of a certain class of completely primary finite rings, Ring and Module Theory, Trends in Mathematics 2010, Springer Basel, pp 83-90.

[9] J. Dutta, D. K. Basnet and R. K. Nath, A note on $n$-centralizer finite rings, An. Stiint. Univ. Al. I. Cuza Iasi. Mat. (N.S.), LXIV (2018), 161-171. 
[10] J. B. Derr, G. F. Orr and P. S. Peck, Noncommutative rings of order $p^{4}$, J. Pure Appl. Algebra, 97 (1994), 109-116.

[11] K. E. Eldridge, Orders for finite noncommutative rings with unity, Amer. Math. Monthly, 75 (1968), 512-514.

[12] B. Fine, Classification of finite rings of order $p^{2}$, Math. Magazine, 66 (1993), 248-252.

[13] R. W. Goldbach and H. L. Claasen, Classification of not commutative rings with identity of order dividing $p^{4}$, Indag. Math., 6 (1995), 167-187.

[14] D. MacHale, Commutativity in finite rings, Amer. Math. Monthly, 83 (1976), 30-32.

[15] R. Raghavendran, A class of finite rings, Compositio Math., 22 (1970), 49-57.

Jutirekha Dutta Department of Mathematical Sciences, Tezpur University, Napaam-784028, Sonitpur, Assam, India.

E-mail: jutirekhadutta@yahoo.com

Dhiren Kumar Basnet Department of Mathematical Sciences, Tezpur University, Napaam784028, Sonitpur, Assam, India.

E-mail: dbasnet@tezu.ernet.in

Rajat Kanti Nath Department of Mathematical Sciences, Tezpur University, Napaam-784028, Sonitpur, Assam, India.

E-mail: rajatkantinath@yahoo.com 